\section{Iatrogenic Cushing Syndrome}

We are presenting three cases of iatrogenic Cushing syndrome in infants, that we came across recently.

The first infant was an 8 month old boy, who was brought to the outpatient for complaints of poor growth and hirsutism. On initial history, the parents categorically denied administration of any medication/'tonic' to the baby. However, when confronted again, after a thorough investigative work-up that failed to reveal any etiology, the mother admitted that she had been giving betamethasone drops to the baby since the age of 1 month, when it was prescribed by a local practioner for upper respiratory tract infection. She was so impressed by the voracious appetite and mood elevation that she continued it till the age of 7 months, and then stopped abruptly as she herself sensed that the baby's poor growth/ hirsuitism could be an adverse effect.

The second infant was a 3-month-old boy who was taking betamethasone drops for the last $2 \frac{1}{2}$ months, again prescribed for some minor respiratory tract illness. In this child the medication was suddenly stopped during a period of stress (child had fever and lethargy for 2 days). This precipitated an adrenal crisis (shock with severe metabolic acidosis, hyperkalemia and hypoglycemia). Child was admitted in the ICU and required stress doses of steroids and other supportive care. He gradually improved and was later discharged on tapering doses of oral steroids.

The third infant was an 8-month boy, who was diagnosed with congenital glaucoma at the age of 3 months. He underwent trabeculectomy at 6 months of age, and was prescribed betamethasone eye drops to prevent post operative inflammation and subconjuctival fibrosis [1]. At presentation (8-months age) the child had cushingoid habitus, hirsutism and poor weight and height growth. His serum cortisol estimation was low $(1.19 \mathrm{mcg} /$ $\mathrm{dl})$, consistent with exogenous steroid overdose.

In the first two cases reported here, glucocorticoids were prescribed without any indication and then continued for inappropriately long durations by the parents, leading to Cushing syndrome. These cases illustrate how things can go awry if there is lack of clear communication between the health caregiver and the patient's family regarding the dose, duration and adverse effects of medication. Easy availability of almost all medications over the counter, as well as medical practice by unqualified persons, predispose to such adverse events. Strong measures are needed to curb nonprescription dispensing by chemists, and practicing of medicine by quacks, who often prescribe steroids as a quick-fix remedy for dubious indications. There is no definitive indication for use of betamethasone oral drops in clinical practice. The policy-makers and the pharmaceutical companies should consider withdrawing this drug from the market.

In the third case, Cushing syndrome occured secondary to topical therapy with steroid eye drops. This is an exceptional event and only four case reports are cited to date in pediatric age [2-5]. This case highlights the fact that small but tangible risk associated with topical steroid therapy should always be kept in mind and all patients on steroids (systemic or topical) should be closely monitored.

In all the three cases parents were explained regarding the need for stress dosing of steroids during any illness for the next 6 months. This is because the suppressed hypothalamic-pituitary-adrenal axis takes time to recover its normal function.

$$
\begin{array}{r}
\text { NishaNT VERMA AND *VANDANA JAIN } \\
\text { *Division of Pediatric Endocrinology and } \\
\text { Department of Pediatrics, } \\
\text { All India Institute of Medical Sciences, New Delhi, India. } \\
\text { drvandanajain@gmail.com }
\end{array}
$$

\section{REFERENCES}

1. Nguyen KD, Lee DA. Effect of steroids and nonsteroidal anti-inflammatory agents on human ocular fibroblast. Invest Ophthalmol Vis Sci. 1992;33:2693-2701.

2. Ozerdem U, Levi L, Cheng L, Song MK, Scher C, Freeman WR. Systemic toxicity of topical and periocular corticosteroid therapy in an 11-year-old male with posterior uveitis. Am J Ophthalmol. 2000;107:240-1.

3. Steelman J, Kappy M. Adrenal suppression and growth retardation from ocular corticosteroids. J Pediatr Ophthalmol Strabismus. 2001;38:177-8.

4. Messina MF, Valenzise M, Aversa S, Arrigo T, Luca FD. Iatrogenic Cushing syndrome caused by ocular glucocorticoids in a child. BMJ Case Reports 2009; Published 1 January 2009; published online 8 May 2009.

5. Romano PE, Traisman HS, Green OC. Fluorinated corticosteroid toxicity in infants. Am J Ophthalmol. 1977;84:249-50. 